\title{
Hydrogenation of $p$-chloronitrobenzene on La-doped NiMoB nanocluster catalysts
}

\author{
Der-Shing Lee, Yu-Wen Chen* \\ Department of Chemical and Materials Engineering, National Central University, Chung-Li 320, Taiwan, China
}

\section{A R T I C L E I N F O}

Article history:

Received 30 March 2013

Accepted 5 June 2013

Published 20 November 2013

\section{Keywords:}

Nickel

Lanthanum boride

Hydrogenation

Chloronitrobenzene

Chloroaniline

\begin{abstract}
A B S T R A C T
Mo and La have been independently reported to be good promoters of NiB nanocluster in the hydrogenation of para-chloronitrobenzene ( $p$-CNB). However, a bi-promoter effect of Mo and La in $\mathrm{NiB}$ catalysts has not previously been investigated. In this work, a series of La-Mo-doped, nanosized $\mathrm{NiB}$ catalysts with different La contents were prepared by chemical reduction and were characterized by X-ray diffraction, transmission electron microscopy, high-resolution transmission electron microscopy, and X-ray photoelectron spectroscopy. The liquid-phase hydrogenation of $p$-CNB was carried out in a batch reactor at $333 \mathrm{~K}, 1.2 \mathrm{MPa} \mathrm{H}_{2}$ pressure and at a spinning rate of $500 \mathrm{r} / \mathrm{min}$. Even doping a small amount of La in NiMoB had a significant effect on its activity and selectivity to $p$-chloroaniline. The addition of an appropriate amount of La suppressed the growth and long-range order of NiMoB and helped the NiMoB maintain its amorphous state. La was determined to be present in its oxide form and to act as a spacer, preventing NiMoB from sintering and aggregation. However, a high amount of La had an opposite effect, and the optimum ratio of La:Ni was 0.2.
\end{abstract}

(C) 2013, Dalian Institute of Chemical Physics, Chinese Academy of Sciences. Published by Elsevier B.V. All rights reserved.

\section{Introduction}

Aromatic haloamines are industrially important for the production of many chemical products, such as dyes, herbicides, pesticides, preservatives, plant growth regulators, medicines, and light-sensitive and nonlinear optical materials. Because of the environmental impact associated with the use of $\mathrm{HCl}$ in Bechamp's reaction, selective hydrogenation of halonitrobenzene to haloaniline over metal catalysts is preferred. Noble metals including Pt, Pd, and Rh have been studied extensively for this reaction, but the application of noble metals is limited because of their low selectivity to haloaniline and their high cost. Raney Ni is also commonly used in the hydrogenation reaction. However, the hydrodehalogenation of halonitrobenzene and aromatic haloamines often occur on Raney $\mathrm{Ni}$, and it suffers the disadvantage of self-igniting.

Nanocatalysts have more surface atoms and a higher con- centration of highly coordinated unsaturated sites compared with conventional catalysts. Amorphous nanoalloy particles have attracted considerable attention because of their interesting intrinsic properties (for example, short-range order, long-range disorder, and high dispersibility) as well as their potential applications (for example, in powder metallurgy and ferrofluids, and as magnetic materials and catalysts). Amorphous nanoalloy particles combine the features of amorphous and ultrafine powders and have several properties that are of interest in catalysis: (1) a large number of surface coordinating unsaturated sites, (2) a lack of crystal defects, and (3) an isotropic, single-phase nature. Nanosized $\mathrm{Ni}$ catalysts modified with $\mathrm{B}$ have been reported to be good catalysts for hydrogenation of nitrobenzene and furfural [1-10]. The catalytic properties were found to be highly dependent upon the preparation method [2-5]. B is able to donate partial electrons to Ni, resulting in electron-enriched $\mathrm{Ni}$, and increases the activity and

* Corresponding author. Tel: +886-3-4227151 ext. 34203; Fax: +886-3-4252296; E-mail: ywchen@ncu.edu.tw DOI: 10.1016/S1872-2067(12)60687-1 | http://www.sciencedirect.com/science/journal/18722067| Chin. J. Catal., Vol. 34, No. 11, November 2013 
thermal stability of the catalyst [11-15]. Adding specific modifiers during preparation is another efficient strategy to improve the catalytic activity.

Nanoalloy particles comprising overlapping areas of amorphous alloy and nanophase materials offer advantageous properties because of the combination of their long-range disordered structure and nanosize. Wang et al. [16] have studied the effects of additives on catalytic activity using the hydrodeoxygenation of phenol as a model reaction. They reported that Co- and La-doped, amorphous NiMoB catalysts exhibit very high catalytic activity. The dopant was shown to exist in various states in the catalyst, such as the elemental, oxide or hydroxide states, with the relative abundance of each state depending strongly on the preparation method. However, the effect of La content was not investigated. It has been reported that La-NiB is much more active than $\mathrm{NiB}$ and the Raney Ni catalyst $[17,18]$. The selectivity of a La-NiB catalyst to the main product ( $p$-chloroaniline, $p$-CAN) was reported to be $>99 \%$ with the promoting effect of La being attributed to its electronic modification of $\mathrm{Ni}[17,18]$.

In a previous study, one of the authors reported that NiMoB is a good catalyst for hydrogenation of $p$-chloronitrobenzene ( $p$-CNB) [6]. The objective of the present study was to investigate the effect of co-doping La and Mo on the catalytic properties of $\mathrm{NiB}$ for the liquid-phase hydrogenation of $p$-CNB to $p$-CAN. The catalysts were characterized by inductively coupled plasma mass spectrometry (ICP-MS), X-ray diffraction (XRD), transmission electron microscopy (TEM), high-resolution TEM (HRTEM), energy dispersive spectroscopy (EDS), and X-ray photoelectron spectroscopy (XPS).

\section{Experimental}

\subsection{Preparation of catalysts}

A series of La-doped NiMoB catalysts were prepared by the chemical reduction method. Nickel acetate, ammonium heptamolybdate, and lanthanum nitrate hexahydrate (> 99.9\%, supplied by Stream Chemicals, Newburyport, MA, USA) were mixed in a methanolic solution (20 mL, $0.1 \mathrm{~mol} / \mathrm{L})$ at room temperature under vigorous stirring for 15 min under a $\mathrm{N}_{2}$ stream to remove $\mathrm{O}_{2}$ from the reaction solution. A solution containing the reduction agent, $\mathrm{NaBH}_{4}(>98 \%$, purchased from Alfa Aesar, $6 \mathrm{~mL}, 1 \mathrm{~mol} / \mathrm{L})$, was then added dropwise with a micro-tubing pump into the precursor salt solution under a $\mathrm{N}_{2}$ stream. The molar ratio of Ni:Mo:B was fixed at 1:0.4:3. An excess of $\mathrm{NaBH}_{4}$ was used to ensure the complete reduction of the metal cations. Su et al. [6] reported that the optimum molar ratio of Mo:Ni was 0.4 . In the present study, the samples with La:Ni molar ratios of $0.02,0.05,0.1,0.2,0.4,0.6,0.7$, and 0.8 were prepared and investigated. After $30 \mathrm{~min}$, the black precipitate was centrifuged and washed with deionized water three times and absolute methanol twice to remove the impurities (i.e., $\mathrm{Na}^{+}$ions and excess boride species). The precipitate was stored in methanol until the time of use. Liu et al. [1,2] reported that using $50 \mathrm{vol} \%$ methanolic solution to prepare the catalyst yielded the smallest particle size and the highest activity. Thus, a 50 vol\% methanolic solvent was used as the medium in this study. According to our previous results, the 50 vol\% methanolic solution results in the preparation environment being at the isoelectric point; that is, the $\mathrm{pH}$ value at which the net electric charge of a molecule in solution is zero. Hence, the as-prepared catalysts would have a smaller particle size under these conditions.

\subsection{Catalyst characterization}

The as-prepared samples were dried under vacuum before characterization.

The elemental composition of the as-prepared catalyst was determined by ICP-MS (PE-SCIEX ELAN 6100 DRC). The sample was digested in a mixture of $1 \mathrm{~mL}$ of $\mathrm{HNO}_{3}$ (65\%), $3 \mathrm{~mL}$ of $\mathrm{HCl}$ (37\%), and $5 \mathrm{~mL}$ of HF (40\%) in a microwave oven. The ICP-MS was connected to a Geolas Excimer laser ablation system, which featured a homogenized $193 \mathrm{~nm}$ ArF laser beam and output energy of $30 \mathrm{~mJ} / \mathrm{cm}^{2}$.

XRD analysis was performed using a Siemens D500 powder diffractometer. The XRD patterns were collected using $\mathrm{Cu} K_{\alpha 1}$ radiation (1.5405 $\AA$ ) at a tube voltage and current of $40 \mathrm{kV}$ and $20 \mathrm{~mA}$, respectively. The sample was prepared as a thin layer on the sample holder and scanned over the range of $2 \theta=$ $20^{\circ}-70^{\circ}$ at a rate of $0.05^{\circ} / \mathrm{s}$.

The morphologies, dispersive effect, and particle sizes of the samples were determined by TEM (Jeol JEM-2000 FX II). A small amount of sample was put into the sample bottle filled with 99.8\% methanol. After agitation under ultrasound for 90 min, one drop of the dispersed slurry was dropped onto the carbon-coated copper mesh (300\#; Ted Pella Inc., Redding, CA, USA) and dried under vacuum overnight.

HRTEM was carried out on a Jeol JEM-2010 instrument combined with an EDS (Oxford, UK). The HRTEM images were recorded digitally by a Gatan slow scan camera (GIF). A very small amount of sample was put into the sample bottle filled with methanol and agitated under ultrasound for $90 \mathrm{~min}$. One drop of the dispersed slurry was dropped onto a carbon-coated copper mesh and dried under vacuum overnight.

The composition and the oxidation state of each element on the surface of the catalyst were studied by XPS on a Thermo VG Scientific Sigma Probe spectrometer. Al $K_{\alpha}$ radiation was used as the excitation source $(h v=1486.6 \mathrm{eV}, 20 \mathrm{kV}, 30 \mathrm{~mA})$. The sample was pressed as a self-supported plate and mounted on the sample cell. It was then degassed in the pretreatment chamber at $343 \mathrm{~K}$ for $2 \mathrm{~h}$ and transferred into the analyzing chamber where the background pressure was lower than $1 \times 10^{-7} \mathrm{~Pa}$. Before XPS analysis, the sample was sputtered using $\mathrm{Ar}^{+}$ions for $15 \mathrm{~min}$ to remove the oxidized species. All binding energies were calibrated using the contaminant carbon $(\mathrm{C} 1 \mathrm{~s}=$ $285 \mathrm{eV}$ ). The XPS spectrum of each element was deconvoluted using a Gaussian-Lorentz curve-fitting program, the background was corrected using a Shirley-type baseline, and the peak type was Gaussian-Lorenz (7:3). The surface composition of each sample was calculated using the corresponding peak areas, and the PHI sensitivity factors were applied in the calculation. It should be noted that for $\mathrm{Ni}$, only the $2 p_{3 / 2}$ subbands 
were studied instead of the $2 p_{1 / 2}$ subbands because the former have a much higher signal-to-noise ratio and provide the same information.

\section{3. $p$-CNB hydrogenation}

The catalytic activities of the samples were tested using the liquid-phase hydrogenation of $p$-CNB. All experiments were carried out in a cylindrical stirred-tank reactor (Parr Instrument Model 4842) with a $160 \mathrm{~mL}$ capacity. A four-bladed pitched impeller was used for effective agitation, and the agitator was connected to an electric motor with a variable speed up to $1700 \mathrm{r} / \mathrm{min}$. A pressure transmitter and an automatic temperature controller were also used. The gases were supplied from cylinders and introduced to the base of the reactor. The reactor was filled with $2 \mathrm{mmol} \mathrm{Ni}$ catalyst and $2.54 \mathrm{~g} p$-CNB (> $99 \%$, Acros, Belgium) in $80 \mathrm{~mL}$ methanol; the concentration of $p$-CNB was $0.2 \mathrm{~mol} / \mathrm{L}$. According to the literature [1,2], methanol is a better reaction medium than ethanol for the hydrogenation reaction. Initially, air was flushed out of the reactor by $\mathrm{H}_{2}$ at room temperature. After the designated temperature (333 K) was reached, $\mathrm{H}_{2}$ (> 99.99\%, Air Products) was fed into the reactor to reach the preset pressure of $1.2 \mathrm{MPa}$, and the stirring speed was fixed at $500 \mathrm{r} / \mathrm{min}$. These conditions were maintained throughout the reaction, with time zero defined as the point at which these conditions were first reached. During the run, the samples were withdrawn periodically (every 10 min) and analyzed by a gas chromatograph equipped with a flame ionization detector with a $3 \mathrm{~m} \times 3.175 \mathrm{~mm}$ stainless steel column packed with 5\% OV-101 on Chromsorb WAW-DMSC (80-100 mesh).

The conversion percentage and selectivity to each product were calculated as follows:

$$
\begin{aligned}
& \text { Conversion }=\left(1-C_{\mathrm{p}-\mathrm{CNB}} /\left(C_{\mathrm{AN}}+C_{\mathrm{NB}}+C_{p-\mathrm{CAN}}+C_{p-\mathrm{CNB}}\right)\right) \times 100 \% \\
& S_{\mathrm{AN}}=C_{\mathrm{AN}} /\left(C_{\mathrm{AN}}+C_{\mathrm{NB}}+C_{p-\mathrm{CAN}}\right) \times 100 \% \\
& S_{\mathrm{NB}}=C_{\mathrm{NB}} /\left(C_{\mathrm{AN}}+C_{\mathrm{NB}}+C_{p-\mathrm{CAN}}\right) \times 100 \% \\
& S_{p \text {-CAN }}=C_{p-\mathrm{CAN}} /\left(C_{\mathrm{AN}}+C_{\mathrm{NB}}+C_{p \text {-CAN }}\right) \times 100 \%
\end{aligned}
$$

where $C_{\mathrm{AN}}, C_{\mathrm{NB}}, C_{p \text {-CAN }}$, and $C_{p \text {-CNB }}$ represent the concentrations of aniline, nitrobenzene, $p$-chloroaniline, and $p$-chloronitrobenzene, respectively.

\section{Results and discussion}

\subsection{ICP-MS}

ICP-MS results of all samples are listed in Table 1 . The real amounts of $\mathrm{Ni}, \mathrm{Mo}$, and La were not the same as the nominal

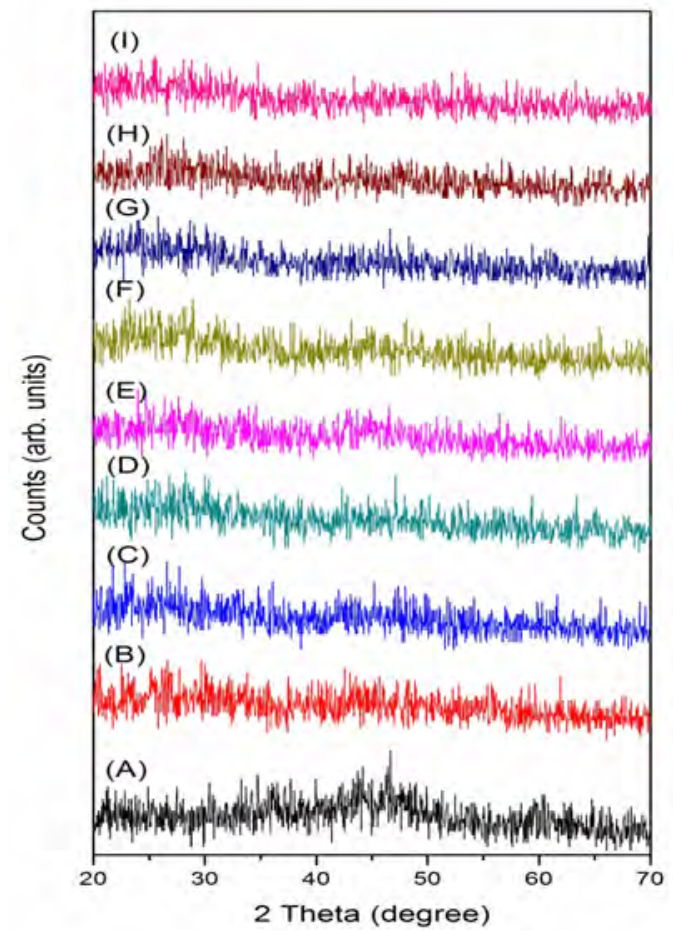

Fig. 1. XRD patterns of La-NiMoB catalysts. (A) NiMoB; (B) 0.02 LaNiMoB; (C) 0.05La-NiMoB; (D) 0.1La-NiMoB; (E) 0.2La-NiMoB; (F) 0.4La-NiMoB; (G) 0.6La-NiMoB; (H) 0.7La-NiMoB; (I) 0.8La-NiMoB.

amounts because of the loss incurred during the preparation process. The results demonstrate that the presence of La influences the surface properties of $\mathrm{Ni}$. When a large amount of $\mathrm{La}$ was added, there was a large loss of each element during preparation.

\section{2. $X R D$}

Figure 1 shows the XRD patterns of the La-NiMoB catalysts. The NiMoB sample showed a broad peak at $2 \theta=4^{\circ}$ and two small peaks at $2 \theta=35^{\circ}$ and $60^{\circ}$. Ni can form several types of compounds with $\mathrm{B}$ such as $\mathrm{NiB}, \mathrm{Ni}_{2} \mathrm{~B}$, and $\mathrm{Ni}_{3} \mathrm{~B}$ [20-24]. However, it was difficult to distinguish which crystalline phase existed in the catalysts because of the low intensities of these peaks. After adding La, the intensities of these peaks further decreased. One can conclude that adding La stabilizes the amorphous structure of $\mathrm{NiMoB}$ and decreases the particle size. When the amount of La was greater than 0.4 , a weak peak around $2 \theta=27^{\circ}$ was observed. Wang et al. [16] ascribed this

\begin{tabular}{|c|c|c|c|c|c|c|}
\hline \multirow{2}{*}{ Catalyst a } & \multicolumn{2}{|c|}{$\mathrm{Ni}(\mathrm{wt} \%)$} & \multicolumn{2}{|c|}{ Mo (wt\%) } & \multicolumn{2}{|c|}{$\mathrm{La}(\mathrm{wt} \%)$} \\
\hline & Nominal & Actual & Nominal & Actual & Nominal & Actual \\
\hline NiMoB & 45.3 & 42.5 & 29.6 & 21.9 & 0 & 0 \\
\hline 0.1La-NiMoB & 40.9 & 35.3 & 26.8 & 19.7 & 9.7 & 8.1 \\
\hline 0.2La-NiMoB & 37.3 & 29.9 & 24.4 & 13.3 & 17.7 & 11.8 \\
\hline 0.4La-NiMoB & 31.7 & 22.6 & 20.7 & 12.6 & 30.0 & 20.6 \\
\hline 0.8La-NiMoB & 24.4 & 10.3 & 15.9 & 6.8 & 46.2 & 19.4 \\
\hline
\end{tabular}
peak to boron oxides. A large amount of La also led to the for-

Table 1

$\mathrm{Ni}, \mathrm{La}$, and Mo amounts in the La-NiMoB catalysts.

a The number in parentheses before La indicates the La:Ni molar ratio. 
mation of larger catalyst particles, possibly due to aggregation of the La species, in particular lanthanum hydroxide.

The effect of La addition on particle size was observed qualitatively during the centrifugation step of catalyst preparation. After adding a small amount of $\mathrm{La}$, the separation of the powder sample from water became more difficult, with many tiny particles suspended in the solution even after a long centrifugation time. However, when the molar ratio of La:Ni was greater than 0.4 , the separation of the catalyst from the solvent was easier. The particle size became smaller after adding a critical amount of $\mathrm{La}$, and we propose that the La species was homogeneously distributed inside the NiMoB and acted as a spacer to suppress the aggregation of NiMoB. When a large amount of La was added, the La species aggregated to form large particles. Differential scanning calorimetry (DSC) was also carried out on the sample, showing that the crystallization temperature of La-NiMoB was $50{ }^{\circ} \mathrm{C}$ higher than that of $\mathrm{NiB}$.

\subsection{TEM, HRTEM, and EDS}

TEM and HRTEM were used to investigate the surface morphology, particle size, and size distribution. Figure 2 shows the TEM images of the modified and unmodified NiMoB catalysts. Each sample was composed of many small particles, and the size of the aggregates was about 30-50 $\mathrm{nm}$. The particles aggregated easily and cannot be clearly observed because of the very strong van der Waals forces [25-31]. Nevertheless, one

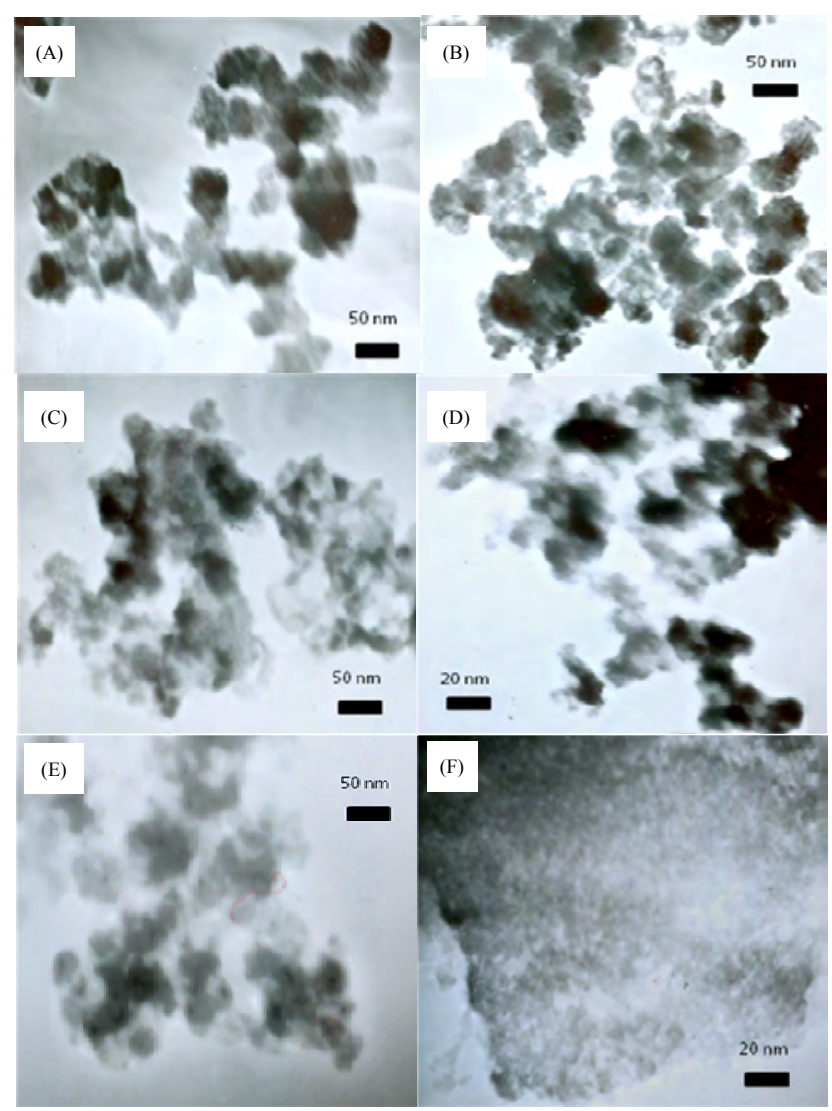

Fig. 2. TEM images of NiMoB (A), 0.02La-NiMoB (B), 0.1 La-NiMoB (C), $0.2 \mathrm{La}-\mathrm{NiMoB}(\mathrm{D}), 0.4 \mathrm{La}-\mathrm{NiMoB}(\mathrm{E})$, and 0.8La-NiMoB (F).
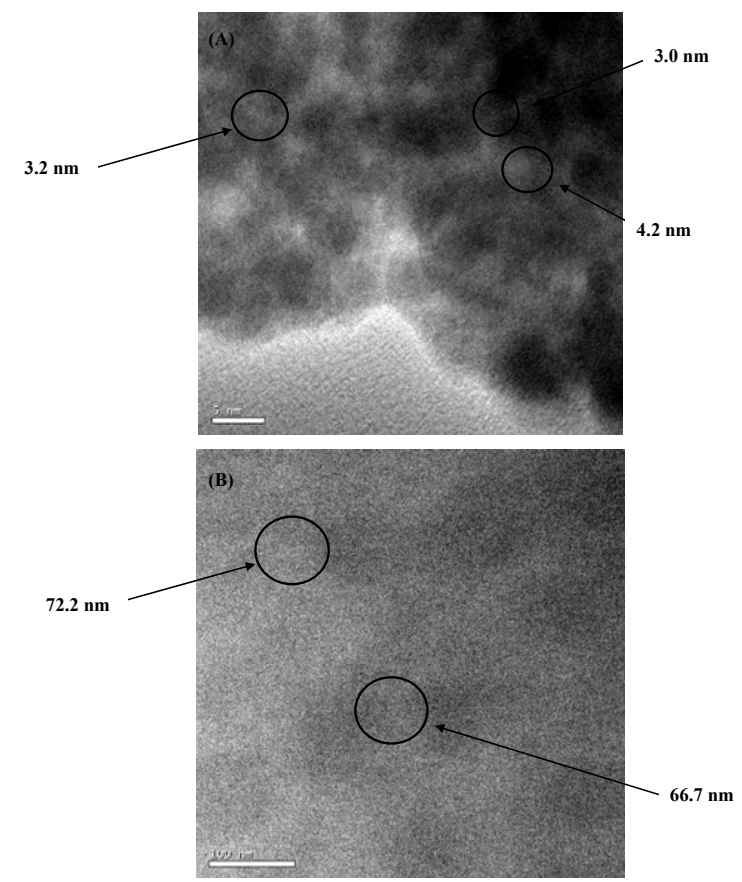

Fig. 3. HRTEM images of $0.2 \mathrm{La}-\mathrm{NiMoB}(\mathrm{A})$ and $0.8 \mathrm{La}-\mathrm{NiMoB}(\mathrm{B})$ catalysts.

can conclude that the La-NiMoB particles were in the nanometer-size range.

HRTEM images of the 0.2 La-NiMoB and 0.8La-NiMoB catalysts are shown in Fig. 3. These images confirm that the La-NiMoB catalysts were composed of many small spherical nanoscale particles, consistent with the literature [24]. As shown in Fig. 3(A), adding a small amount of La decreased the particle size to 3-4 $\mathrm{nm}$. When a large amount of La was added to the NiMoB catalyst, the particle size increased to $\approx 70 \mathrm{~nm}$, as shown in Fig. 3(B). The boundaries of the particles were not clear even at a magnification factor of $5 \times 10^{5}$, and the crystalline lattice fringes were not observed in the HRTEM images. This confirms that these materials were amorphous, which is in accordance with the XRD results and previous studies.

Figure 4 shows EDS spectra of $0.2 \mathrm{La}-\mathrm{NiMoB}$ acquired at one position on the surface of this sample. $B$ was not found because of the poor sensitivity of the EDS technique to this light element. When compared with the ICP-MS results, it can be concluded that the amount of $\mathrm{Ni}$ on the surface was greater than that in the bulk, demonstrating Ni surface enrichment.

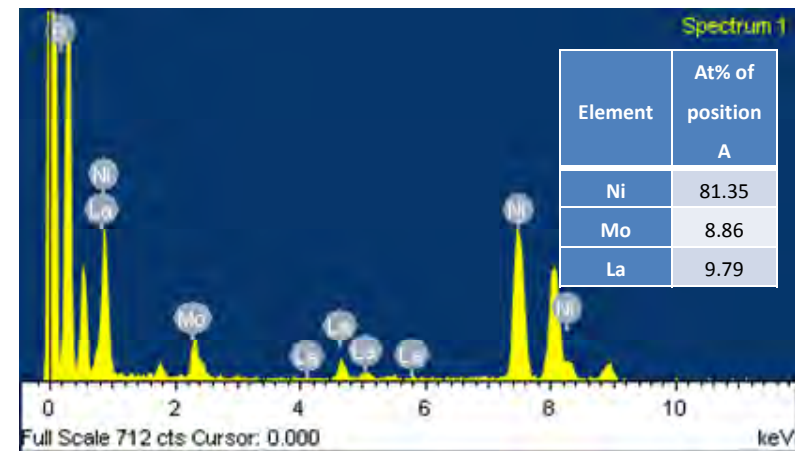

Fig. 4. EDS spectra of $0.2 \mathrm{La}-\mathrm{NiMoB}$ and the corresponding composition. 


\subsection{XPS}

The compositions and electronic structures of each species on the surface of the samples were determined by XPS analysis. The XPS spectra of Ni $2 p$, Mo $3 d$, La $4 d$, and B $1 s$ are shown in Figs. 5-9. All spectra were deconvoluted, and the amount of each state was calculated based on the corresponding peak area. As shown in Figs. 5 and 7, the Ni peak was negatively shifted upon addition of La. The peaks of B, Mo, and La exhibited positive shifts with increasing La concentration. This result indicates that $\mathrm{Ni}, \mathrm{Mo}$, La, and B interacted chemically with each other [24].

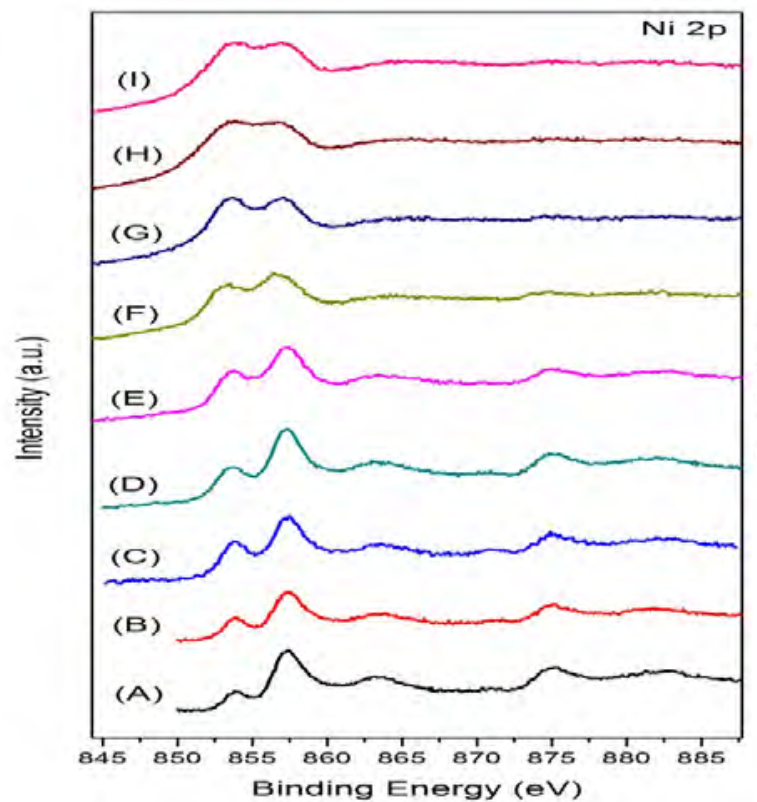

The Ni $2 p_{3 / 2}$ binding energies were 854.1 and $857.2,854.1$ and 857.3, 853.8 and 857.3, 853.7 and 857.3, 853.6 and 857.4, 852.5 and 857.2, 853.2 and 857.0, 853.2 and 857.4, 853.0 and 857.5, and $852.9 \mathrm{eV}$, for the NiMoB, 0.02La-, 0.05La-, 0.1La-, 0.2La-, 0.4La-, 0.6La-, 0.7La-, and 0.8La-NiMoB samples, respectively. These are ascribed to metallic Ni [32]. The peak at $\approx 861.3 \mathrm{eV}$ is ascribed to nickel oxide (simply denoted as $\mathrm{Ni}^{2+}$; similar notations are also used for the other elements). After adding La, the binding energy was lower compared with the unmodified $\mathrm{NiMoB}$ catalyst, indicating that the $\mathrm{Ni}^{0}$ was electron-enriched. 0.2La-NiMoB had the largest negative shift and was therefore the most electron-rich catalyst.

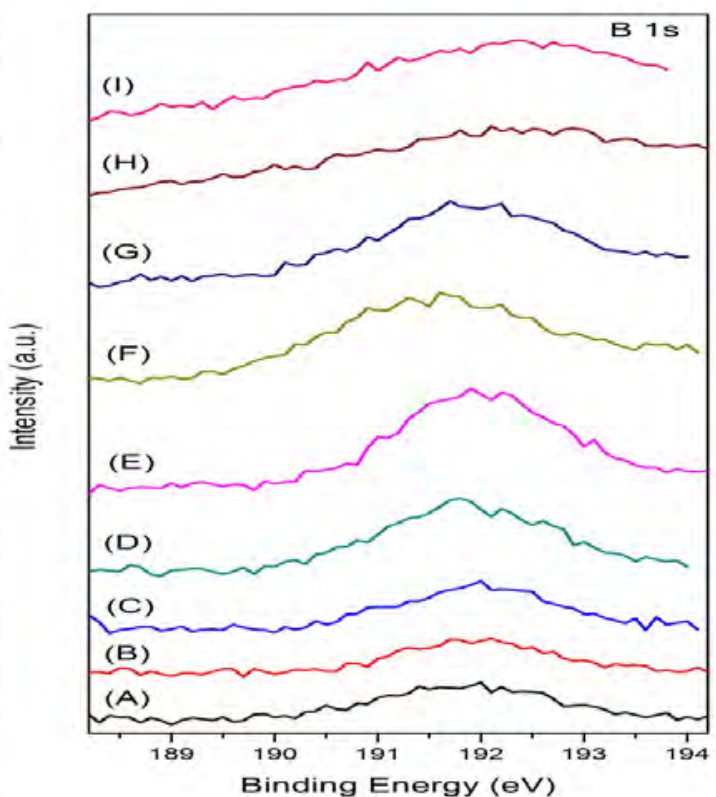

Fig. 5. Ni $2 p$ and B $1 s$ XPS spectra of La-NiMoB catalysts. (A) NiMoB; (B) 0.02La-NiMoB; (C) 0.05La-NiMoB; (D) 0.1La-NiMoB; (E) 0.2La-NiMoB; (F) 0.4La-NiMoB; (G) 0.6La-NiMoB; (H) 0.7La-NiMoB; (I) 0.8La-NiMoB.
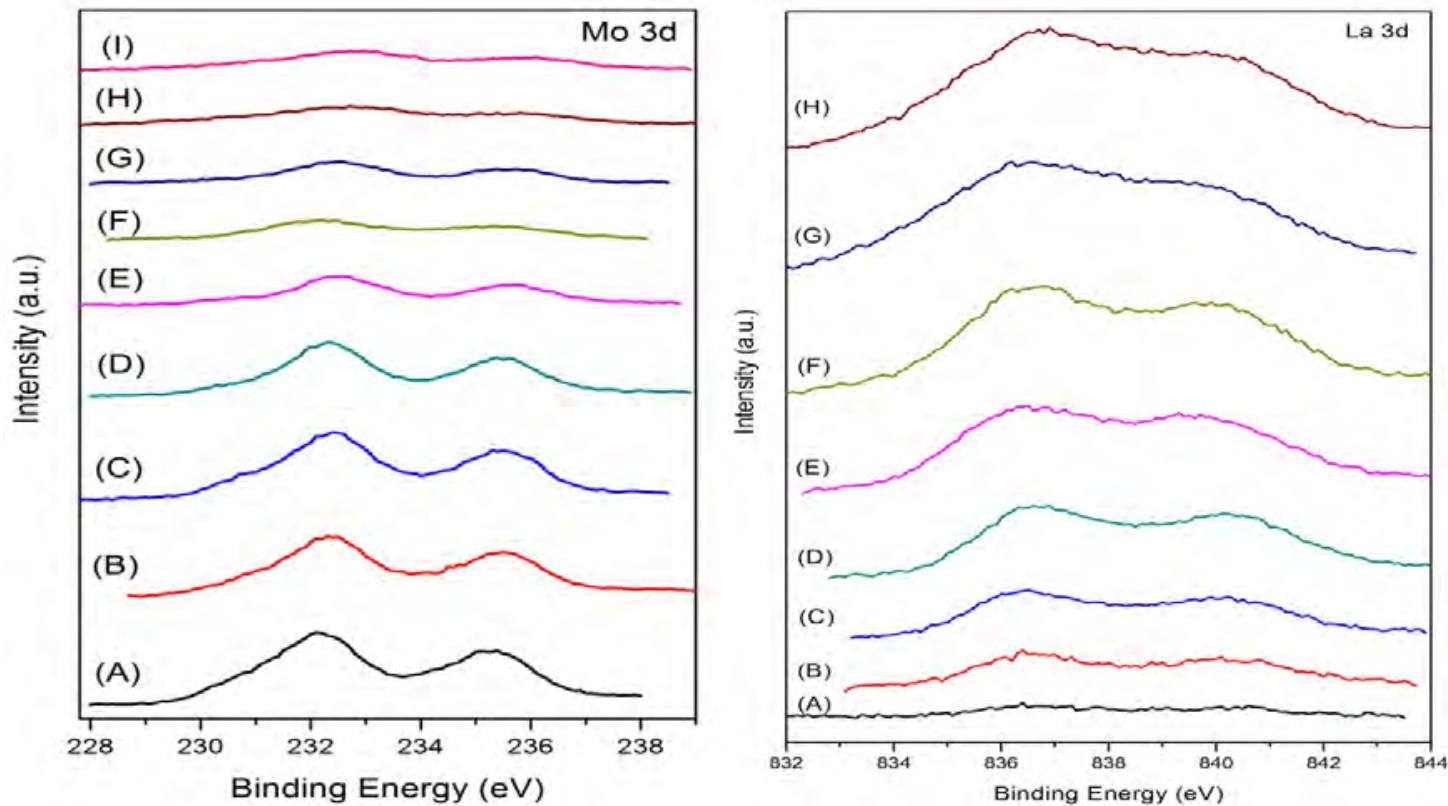

Fig. 6. Mo $3 d$ and La $3 d$ XPS spectra of La-NiMoB catalysts. (A) NiMoB; (B) 0.02La-NiMoB; (C) 0.05La-NiMoB; (D) 0.1La-NiMoB; (E) 0.2La-NiMoB; (F) 0.4La-NiMoB; (G) 0.6La-NiMoB; (H) 0.7La-NiMoB; (I) 0.8La-NiMoB. 


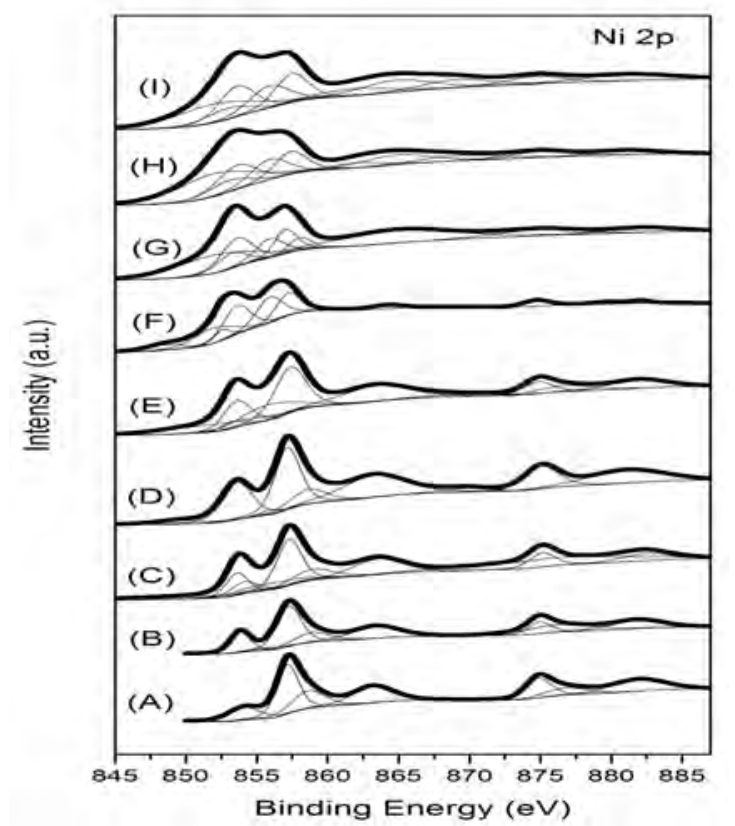

Fig. 7. Ni $2 p$ XPS spectra of La-NiMoB catalysts. (A) NiMoB; (B) 0.02La-NiMoB; (C) 0.05La-NiMoB; (D) 0.1 La-NiMoB; (E) 0.2La-NiMoB; (F) 0.4La-NiMoB; (G) 0.6La-NiMoB; (H) 0.7La-NiMoB; (I) 0.8La-NiMoB.

As shown in Fig. 5, there were two peaks in the $\mathrm{B} 1 s$ spectra. Both $\mathrm{B}$ species were present in alloyed $\mathrm{B}$ and $\mathrm{B}_{2} \mathrm{O}_{3}$. Alloyed $\mathrm{B}$ can donate partial electrons to $\mathrm{Ni}$, leading to an electron enrichment of Ni. The peak at $\approx 187.1 \mathrm{eV}$ is attributed to elemental $\mathrm{B}$, and the peak at $\approx 192.5 \mathrm{eV}$ is attributed to $\mathrm{B}^{3+}$. The binding energy of elemental $B$ would increase to some extent when it is in an alloy. It should be noted that almost all B content in the catalyst was in oxidized states. The binding energy of B $1 \mathrm{~s}$ was assigned to the peaks at 187.3, 187.5, 187.7, 187.5, 189.1, 187.4, 189.9, 190.2, and $190.6 \mathrm{eV}$ for the NiMoB, 0.02La-, $0.05 \mathrm{La}-, 0.1 \mathrm{La}-, 0.2 \mathrm{La}-, 0.4 \mathrm{La}-, 0.6 \mathrm{La}-, 0.7 \mathrm{La}-$, and $0.8 \mathrm{La}-\mathrm{NiMoB}$ samples, respectively. The binding energy of elemental $\mathrm{B}$ is $187.1 \mathrm{eV}$. After adding La, the binding energy of $\mathrm{B}^{0}$ was positively shifted by $0.3-3.5 \mathrm{eV}$, indicating that partial electrons transferred from alloyed $\mathrm{B}$ to metallic $\mathrm{Ni}$ in the La-modified NiMoB catalyst. The positive shift of the B peak increased as the La content increased. Because of the greater atomic weight of $\mathrm{Ni}$ compared with B, the binding energy shift for the Ni species was not discernible [11].

According to the literature [9], the following reactions take place during preparation of the catalysts:

$$
\begin{gathered}
\mathrm{BH}_{4}^{-}+2 \mathrm{H}_{2} \mathrm{O} \rightarrow \mathrm{BO}^{2-}+4 \mathrm{H}_{2} \\
\mathrm{BH}_{4}^{-}+4 \mathrm{Ni}^{2+}+8 \mathrm{OH}^{-} \rightarrow 4 \mathrm{Ni}+\mathrm{BO}^{2-}+6 \mathrm{H}_{2} \mathrm{O} \\
\mathrm{BH}_{4}^{-}+\mathrm{H}_{2} \mathrm{O} \rightarrow \mathrm{B}+\mathrm{OH}^{-}+2.5 \mathrm{H}_{2}
\end{gathered}
$$

In addition to the above reactions, Mo and La cations can also be reduced by $\mathrm{BH}_{4}{ }^{-}$. As a result, Mo and $\mathrm{La}$ are present in both their elemental and oxide states. Because of the different reduction potentials of the precursors and the different activation energies of these reactions, the rates of the above reactions and their contributions to the overall reaction and to the final catalyst are different. Such differences could influence the chemical state of Mo and La, as well as the boron content in the catalyst.

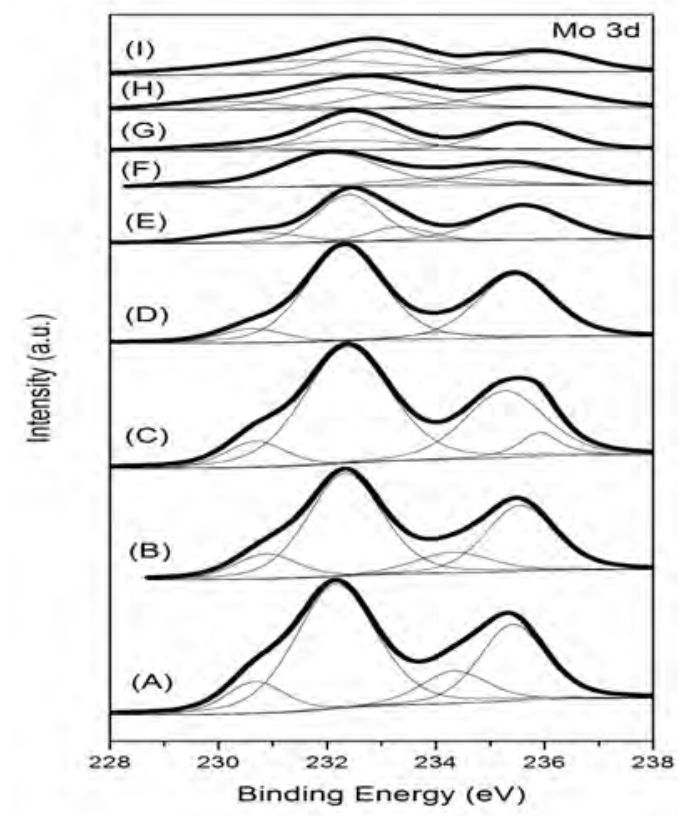

Fig. 8. Mo $3 d$ XPS spectra of La-NiMoB catalysts. (A) NiMoB; (B) 0.02La-NiMoB; (C) 0.05La-NiMoB; (D) 0.1La-NiMoB; (E) 0.2 La-NiMoB; (F) 0.4La-NiMoB; (G) 0.6La-NiMoB; (H) 0.7La-NiMoB; (I) 0.8La-NiMoB.

Figure 8 displays the deconvolution of the XPS Mo $3 d_{5 / 2}$ peak for the La-NiMoB catalyst powder. The binding energy of the standard state of Mo $3 d_{5 / 2}$ is $228 \mathrm{eV}$. The Mo cation is not easily reduced and was consequently present in the hydroxide state. The binding energies of Mo $3 d_{5 / 2}$ were 228.9, 230.7, 230.6, 230.5, 231.1, 229.3, 230.2, 229.8, and $227.3 \mathrm{eV}$ for the NiMoB and $0.02 \mathrm{La}-, 0.05 \mathrm{La}-, 0.1 \mathrm{La}-, 0.2 \mathrm{La}-, 0.4 \mathrm{La}-, 0.6 \mathrm{La}-$, $0.7 \mathrm{La}-$, and $0.8 \mathrm{La}-\mathrm{NiMoB}$ samples, respectively. The binding energies of $\mathrm{Mo}^{0}$ measured are therefore higher than the standard value of $228.0 \mathrm{eV}$. After adding La, the binding energy of $\mathrm{Mo}^{0}$ was positively shifted, indicating that electrons were donated from $\mathrm{Mo}^{0}$ to $\mathrm{Ni}^{0}$, making the $\mathrm{Ni}^{0}$ electron-enriched. It is worth mentioning that the binding energy of $\mathrm{Mo}^{0}$ is negatively shifted in the 0.8La-NiMoB sample. Rather than donating electrons to $\mathrm{Ni}^{0}$, La in this case accepts electrons from other electron donors. This results in $\mathrm{Ni}^{0}$ being less electron-rich, which may be the cause of its poor activity.

Figure 9 displays the XPS La $3 d_{5 / 2}$ peak analysis for the La-NiMoB sample. As shown in Table 2, about half of the La was reduced by $\mathrm{NaBH}_{4}$ with the remainder present in the hydroxide state. The binding energy of the standard state of La $3 d_{5 / 2}$ is 836 $\mathrm{eV}$. The binding energies of La were determined to be 836.8, 836.5, 836.5, 836.7, 836.4, 836.6, 836.5, and $836.7 \mathrm{eV}$ for the $0.02 \mathrm{La}-, 0.05 \mathrm{La}-, 0.1 \mathrm{La}-, 0.2 \mathrm{La}-, 0.4 \mathrm{La}-, 0.6 \mathrm{La}-, 0.7 \mathrm{La}-$, and $0.8 \mathrm{La}-\mathrm{NiMoB}$ samples, respectively. The binding energies of $\mathrm{La}^{0}$ measured were therefore higher than the standard value of 836 $\mathrm{eV}$, indicating that $\mathrm{La}^{0}$ donated partial electrons to $\mathrm{Ni}^{0}$. The peak around $838.3 \mathrm{eV}$ was ascribed to lanthanum hydroxide. We propose that lanthanum hydroxide acts as a spacer keeping the NiMoB particles nanosized and with an amorphous structure while suppressing aggregation.

The relative amounts of the elemental and oxidized states of each species in the catalysts are shown in Table 2 (the numbers 


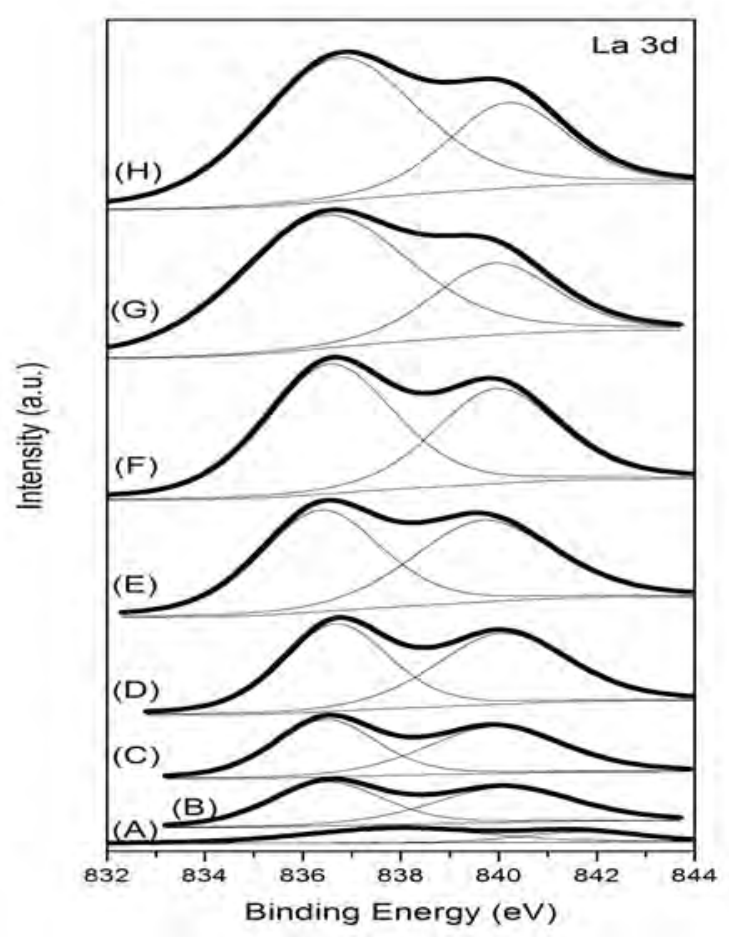

Fig. 9. XPS La $3 d$ spectra of La-NiMoB catalysts. (A) 0.02La-NiMoB; (B) 0.05La-NiMoB; (C) 0.1La-NiMoB; (D) 0.2La-NiMoB; (E) 0.4La-NiMoB; (F) 0.6La-NiMoB; (G) 0.7La-NiMoB; (H) 0.8La-NiMoB.

in parentheses). More than half of the $\mathrm{Ni}$ and about half of the La were in the elemental state, whereas Mo was mainly in the form of hydroxides. The content of $\mathrm{Ni}^{0}$ and $\mathrm{Ni}^{2+}$ was calculated from the corresponding peak areas, and the results are listed in Table 2. It is known that hydrogen molecules adsorb to the $\mathrm{Ni}^{0}$ sites and are activated before the hydrogenation reaction. Therefore, the amount of $\mathrm{Ni}^{0}$ on the surface of the catalyst is crucial to its catalytic activity in the hydrogenation reaction. $0.2 \mathrm{La}-\mathrm{NiMoB}$ had the highest surface $\mathrm{Ni}^{0}$ content, which is an important determinant of its high catalytic activity for $p$-CNB hydrogenation. These results are consistent with the literature [24]. Although the content of alloyed B decreased with La doping in the NiMoB catalysts, this did not affect the activity. Adding La reduced the content of $\mathrm{B}$ on the surface, but $\mathrm{La}^{0}$ and $\mathrm{Mo}^{0}$ were able to donate electrons to the $\mathrm{Ni}^{0}$ active sites. We reported in our previous study that the catalyst was more active with a greater amount of molybdenum hydroxide species present. The addition of La not only maintains a constant surface content of $\mathrm{Ni}$, it also increases the content of molybdenum hydroxide species on the catalyst surface, which can disperse the NiMoB particles and increase the number of unsaturated sites.

The surface compositions of the catalysts are listed in Table 2. For the $0.2 \mathrm{La}-\mathrm{NiMoB}$ catalyst, the surface content of $\mathrm{Ni}$ was $67.8 \%$ with $87.9 \%$ of the $\mathrm{Ni}$ in the elemental state. Thus, there was $60 \%$ elemental $\mathrm{Ni}$ on the catalyst surface, which is much greater than on the NiMoB catalyst. The Mo content was $16.0 \%$, consisting of $2.0 \%$ elemental Mo and $14.0 \%$ molybdenum hydroxide. The La content was $11.1 \%$, consisting of $6.0 \%$ elemental La and 6.0\% lanthanum hydroxide.

\section{5. $p$-CNB hydrogenation}

The catalytic activities were measured for the liquid-phase hydrogenation of $p$-CNB at $1.2 \mathrm{MPa} \mathrm{H}_{2}$ partial pressure and a stirring speed of $500 \mathrm{r} / \mathrm{min}$. The reaction was run at 333 and $353 \mathrm{~K}$. The main product was $p$-CAN, and there were two by-products nitrobenzene (NB) and aniline (AN). NB was generated from the $p$-CNB hydrodehalogenation, whereas AN formed via further hydrogenation of NB and/or hydrodehalogenation of $p$-CAN [33-42]. The results of $p$-CNB hydrogenation at $353 \mathrm{~K}$ are shown in Figure 10 and Table 3. The conversion reached $100 \%$ in $60 \mathrm{~min}$ for most of the samples, and the selectivity of all La-doped samples exceeded 90\%. Because all samples were very active at $353 \mathrm{~K}$, it is difficult to differentiate them at this temperature. Therefore, the temperature was lowered to $333 \mathrm{~K}$ to accentuate the differences between samples. Figure 11 shows the conversion of $p$-CNB and the selectiv-

Table 2

Surface compositions of the La-NiMoB catalysts.

\begin{tabular}{|c|c|c|c|c|c|c|c|c|c|}
\hline \multirow{2}{*}{ Catalyst } & \multirow{2}{*}{$\begin{array}{l}\text { Surface composition } \\
\text { (atomic ratio) }\end{array}$} & \multicolumn{2}{|c|}{$\mathrm{Ni} 2 p_{3 / 2}$} & \multicolumn{2}{|c|}{ Mo $3 d_{5 / 2}$} & \multicolumn{2}{|c|}{ La $3 d_{5 / 2}$} & \multicolumn{2}{|c|}{ B $1 s$} \\
\hline & & $\mathrm{Ni}^{0}$ & $\mathrm{Ni}^{2+}$ & $\mathrm{Mo}^{0}$ & $\mathrm{Mo}^{n+}$ & $\mathrm{La}^{0}$ & $\mathrm{La}^{n+}$ & $\mathrm{B}^{0}$ & $\mathrm{~B}^{3+}$ \\
\hline $\mathrm{NiMoB}$ & $\mathrm{Ni}_{42.1} \mathrm{Mo}_{31.6} \mathrm{~B}_{26.3}$ & $\begin{array}{c}0.28 \\
(68.6 \%)\end{array}$ & $\begin{array}{c}0.13 \\
(31.4 \%)\end{array}$ & $\begin{array}{c}0.19 \\
(60.1 \%)\end{array}$ & $\begin{array}{c}0.13 \\
(39.9 \%)\end{array}$ & - & - & $\begin{array}{c}0.07 \\
(25.4 \%)\end{array}$ & $\begin{array}{c}0.20 \\
(74.6 \%)\end{array}$ \\
\hline 0.02La-NiMoB & $\mathrm{Ni}_{57.3} \mathrm{Mo}_{37.4} \mathrm{La}_{2.0} \mathrm{~B}_{3.4}$ & $\begin{array}{c}0.41 \\
(71.5 \%)\end{array}$ & $\begin{array}{c}0.16 \\
(28.5 \%)\end{array}$ & $\begin{array}{c}0.08 \\
(20.8 \%\end{array}$ & $\begin{array}{c}0.30 \\
(79.2 \%)\end{array}$ & $\begin{array}{c}0.01 \\
(68.2 \%)\end{array}$ & $\begin{array}{c}0.01 \\
(31.8 \%)\end{array}$ & $\begin{array}{c}0.01 \\
(33.1 \%)\end{array}$ & $\begin{array}{c}0.02 \\
(66.9 \%)\end{array}$ \\
\hline 0.05La-NiMoB & $\mathrm{Ni}_{59.7} \mathrm{Mo}_{32.9} \mathrm{La}_{4.0} \mathrm{~B}_{3.3}$ & $\begin{array}{c}0.43 \\
(75.8 \%)\end{array}$ & $\begin{array}{c}0.14 \\
(24.2 \%)\end{array}$ & $\begin{array}{c}0.09 \\
(15.0 \%)\end{array}$ & $\begin{array}{c}0.28 \\
(85.0 \%)\end{array}$ & $\begin{array}{c}0.02 \\
(50.0 \%)\end{array}$ & $\begin{array}{c}0.02 \\
(50.0 \%)\end{array}$ & $\begin{array}{c}0.01 \\
(26.4 \%)\end{array}$ & $\begin{array}{c}0.02 \\
(73.6 \%)\end{array}$ \\
\hline 0.1 La-NiMoB & $\mathrm{Ni}_{66.4} \mathrm{Mo}_{24.5} \mathrm{La}_{5.6} \mathrm{~B}_{3.4}$ & $\begin{array}{c}0.50 \\
(75.2 \%)\end{array}$ & $\begin{array}{c}0.16 \\
(24.8 \%)\end{array}$ & $\begin{array}{c}0.06 \\
(23.7 \%)\end{array}$ & $\begin{array}{c}0.19 \\
(76.3 \%)\end{array}$ & $\begin{array}{c}0.03 \\
(51.0 \%)\end{array}$ & $\begin{array}{c}0.03 \\
(49.0 \%)\end{array}$ & $\begin{array}{c}0.00 \\
(13.3 \%)\end{array}$ & $\begin{array}{c}0.03 \\
(86.7 \%)\end{array}$ \\
\hline 0.2La-NiMoB & $\mathrm{Ni}_{67.8} \mathrm{Mo}_{16.0} \mathrm{La}_{11.1} \mathrm{~B}_{5.1}$ & $\begin{array}{c}0.60 \\
(87.9 \%)\end{array}$ & $\begin{array}{c}0.08 \\
(12.1 \%)\end{array}$ & $\begin{array}{c}0.02 \\
(9.9 \%)\end{array}$ & $\begin{array}{c}0.14 \\
(90.1 \%)\end{array}$ & $\begin{array}{c}0.06 \\
(48.1 \%)\end{array}$ & $\begin{array}{c}0.06 \\
(51.9 \%)\end{array}$ & $\begin{array}{c}0.00 \\
(4.6 \%)\end{array}$ & $\begin{array}{c}0.04 \\
(95.4 \%)\end{array}$ \\
\hline 0.4La-NiMoB & $\mathrm{Ni}_{64.2} \mathrm{Mo}_{12.4} \mathrm{La}_{17.3} \mathrm{~B}_{6.0}$ & $\begin{array}{c}0.43 \\
(67.4 \%)\end{array}$ & $\begin{array}{c}0.21 \\
(32.6 \%)\end{array}$ & $\begin{array}{c}0.02 \\
(17.0 \%)\end{array}$ & $\begin{array}{c}0.10 \\
(83.0 \%)\end{array}$ & $\begin{array}{c}0.09 \\
(52.0 \%)\end{array}$ & $\begin{array}{c}0.08 \\
(48.0 \%)\end{array}$ & $\begin{array}{c}0.00 \\
(1.0 \%)\end{array}$ & $\begin{array}{c}0.06 \\
(99.0 \%)\end{array}$ \\
\hline 0.6La-NiMoB & $\mathrm{Ni}_{56.9} \mathrm{Mo}_{14.0} \mathrm{La}_{24.1} \mathrm{~B}_{5.0}$ & $\begin{array}{c}0.29 \\
(50.4 \%)\end{array}$ & $\begin{array}{c}0.28 \\
(49.6 \%)\end{array}$ & $\begin{array}{c}0.06 \\
(45.4 \%)\end{array}$ & $\begin{array}{c}0.08 \\
(54.6 \%)\end{array}$ & $\begin{array}{c}0.14 \\
(58.9 \%)\end{array}$ & $\begin{array}{c}0.10 \\
(41.1 \%)\end{array}$ & $\begin{array}{c}0.01 \\
(21.3 \%)\end{array}$ & $\begin{array}{c}0.04 \\
(78.7 \%)\end{array}$ \\
\hline 0.7La-NiMoB & $\mathrm{Ni}_{53.5} \mathrm{Mo}_{16.0} \mathrm{La}_{26.4} \mathrm{~B}_{4.1}$ & $\begin{array}{c}0.38 \\
(70.4 \%)\end{array}$ & $\begin{array}{c}0.16 \\
(29.6 \%)\end{array}$ & $\begin{array}{c}0.06 \\
(37.2 \%)\end{array}$ & $\begin{array}{c}0.10 \\
(62.8 \%)\end{array}$ & $\begin{array}{c}0.19 \\
(72.0 \%)\end{array}$ & $\begin{array}{c}0.07 \\
(28.0 \%)\end{array}$ & $\begin{array}{c}0.02 \\
(55.3 \%)\end{array}$ & $\begin{array}{c}0.02 \\
(44.7 \%)\end{array}$ \\
\hline 0.8La-NiMoB & $\mathrm{Ni}_{60.4} \mathrm{Mo}_{11.5} \mathrm{La}_{15.2} \mathrm{~B}_{4.3}$ & $\begin{array}{c}0.41 \\
(67.9 \%)\end{array}$ & $\begin{array}{c}0.19 \\
(32.1 \%)\end{array}$ & $\begin{array}{c}0.06 \\
(40.1 \%)\end{array}$ & $\begin{array}{c}0.08 \\
(59.9 \%)\end{array}$ & $\begin{array}{c}0.15 \\
(68.9 \%)\end{array}$ & $\begin{array}{c}0.07 \\
(31.1 \%)\end{array}$ & $\begin{array}{c}0.02 \\
(52.8 \%)\end{array}$ & $\begin{array}{c}0.02 \\
(47.2 \%)\end{array}$ \\
\hline
\end{tabular}



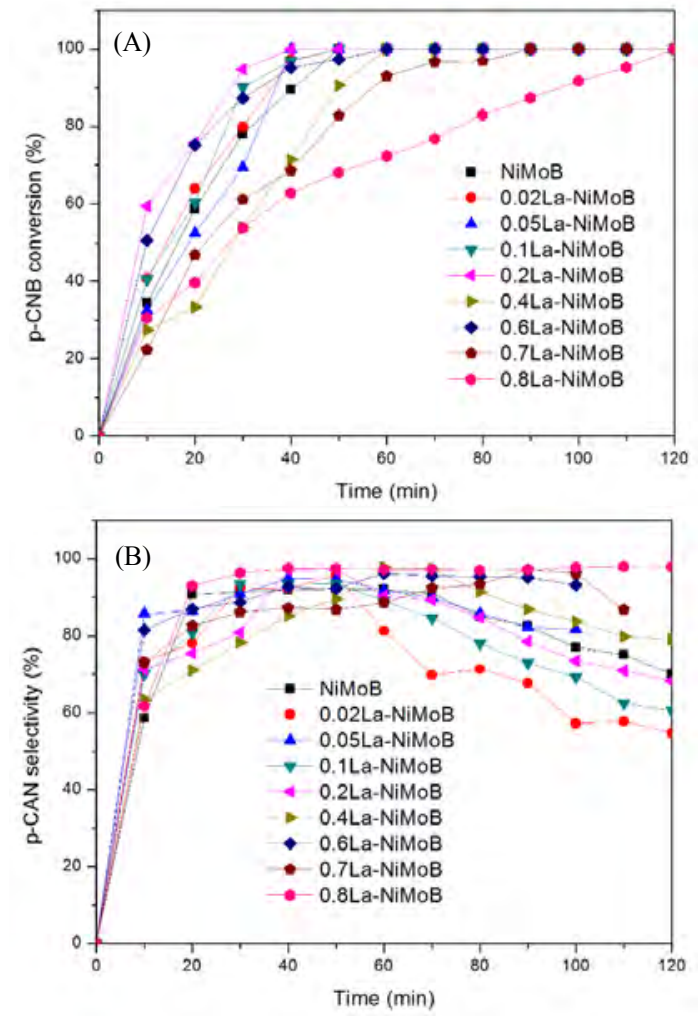

Fig. 10. $p$-CNB conversion (A) and $p$-CAN selectivity (B) as functions of reaction time on La-NiMoB catalysts.

ity to $p$-CAN over these catalysts. The yields of $p$-CAN, NB, and AN are presented in Fig. 10(C), (D), and (E), respectively. In each case, the concentration of NB increased in the first $20 \mathrm{~min}$ and then decreased and converted to AN. Finally, the concentration of NB approached zero. There are two possible explana-
Table 3

The effect of La content on the hydrogenation of $p$-CNB over La-NiMoB catalysts at $353 \mathrm{~K}$.

\begin{tabular}{lcccc}
\hline \multirow{2}{*}{ Catalyst } & \multirow{2}{*}{$\begin{array}{c}\text { Reaction time (min) } \\
\text { at 100\% conversion }\end{array}$} & \multicolumn{3}{c}{ Selectivity (\%) } \\
\cline { 3 - 5 } & 49.2 & 92.2 & 7.8 & 0 \\
\hline NiMoB & 47.6 & 95.8 & 4.2 & 0 \\
0.02 La-NiMoB & 41.0 & 94.9 & 5.1 & 0 \\
0.05La-NiMoB & 48.7 & 93.4 & 6.6 & 0 \\
0.1 La-NiMoB & 38.1 & 97.2 & 2.8 & 0 \\
0.2La-NiMoB & 58.8 & 97.8 & 2.2 & 0 \\
0.4La-NiMoB & 60.3 & 96.2 & 3.8 & 0 \\
0.6 La-NiMoB & 93.0 & 97.2 & 2.8 & 0 \\
0.7La-NiMoB & 120.5 & 98.0 & 2.0 & 0 \\
0.8La-NiMoB &
\end{tabular}

Reaction conditions: $\mathrm{H}_{2} 1.2 \mathrm{MPa}, 353 \mathrm{~K}$, absolute methanol solvent, 500 $\mathrm{r} / \mathrm{min}, 0.2 \mathrm{mmol} \mathrm{Ni}$ catalyst.

p-CAN: $p$-chloroaniline; AN: aniline; NB: nitrobenzene.

tions for these observations: (i) $p$-CNB only followed path (1) in the first $20 \mathrm{~min}$, and (ii) the rate of hydrogenation for path (3) was faster than that of path (1). However, an increasing rate of AN formation was not obvious, thus the possibility of the latter case is low. It is worth mentioning that when the molar ratio of La:Ni was greater than 0.6 , no NB was observed during the reaction.

As demonstrated in Fig. 11, the catalysts doped with an appropriate amount of La showed superior performance to the unmodified catalyst. Although La can increase the reaction rate, a high La content results in a decrease in activity. It has been reported that $\mathrm{B}^{0}, \mathrm{Mo}^{0}$, and $\mathrm{La}^{0}$ are not the active sites for the hydrogenation of $p$-CNB; rather, only $\mathrm{Ni}^{0}$ acts as an active site $[4,24]$. The surface concentration and electron density of $\mathrm{Ni}^{0}$ are the most important factors that determine the rate of reaction. A higher $\mathrm{Ni}^{0}$ content would favor the adsorption of $\mathrm{H}_{2}$ molecules. According to the XPS analysis, all prepared samples
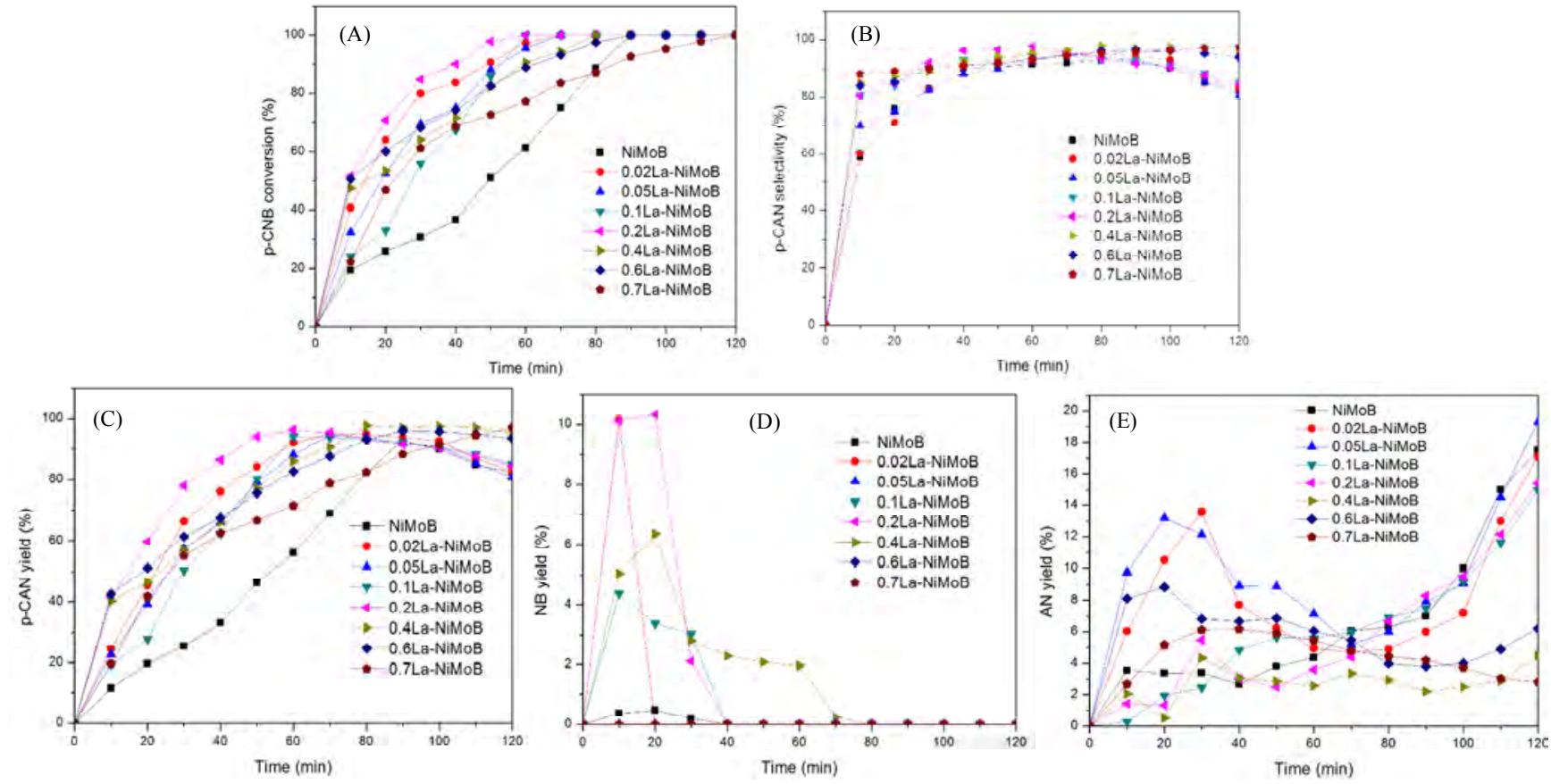

Fig. 11. $p$-CNB conversion (A), $p$-CAN selectivity (B), $p$-CAN yield (C), NB yield (D), and AN yield (E) as functions of reaction time on La-NiMoB catalysts. 
had high densities of elemental Ni. Alloyed B, elemental Mo, and elemental La could donate partial electrons to $\mathrm{Ni}$, making $\mathrm{Ni}$ more electron-rich and B, Mo and La more electron-deficient. Higher electron density at the $\mathrm{Ni}$ active sites can facilitate the formation of $\mathrm{H}^{-}$species [43], which may promote $p$-CNB hydrogenation. Increasing the electron density of $\mathrm{Ni}$ is favorable for activating the adsorbed $-\mathrm{NO}_{2}$ group through a back donation of electrons from the vacant $d$-orbital of $\mathrm{Ni}[15,43]$, which would result in a high rate of adsorption of $p$-CNB at the active elemental $\mathrm{Ni}$ sites. With the addition of La, the binding energy of Ni shifted to a lower value, indicating that Ni accepted electrons from other elements. An appropriate amount of La can also disperse the $\mathrm{Ni}$ active sites and retard aggregation of the NiMoB catalysts. This makes the Ni surface highly unsaturated, favoring $p$-CNB hydrogenation.

The time-conversion curves showed that the reaction was first-order with respect to the $p$-CNB concentration. The activities decreased in the order $0.2 \mathrm{La}-\mathrm{NiMoB}>0.1 \mathrm{La}-\mathrm{NiMoB}>$ $0.02 \mathrm{La}-\mathrm{NiMoB}>0.05 \mathrm{La}-\mathrm{NiMoB}>0.4 \mathrm{La}-\mathrm{NiMoB}>0.6 \mathrm{La}-\mathrm{NiMoB}$ $>$ NiMoB > 0.7La-NiMoB > 0.8La-NiMoB. 0.2La-NiMoB had the highest reaction rate, achieving $50 \%$ conversion within $10 \mathrm{~min}$. The addition of La in low La:Ni ratios promoted the reaction; however, a high La:Ni ratio (>0.7) resulted in a decrease in activity.

Figure 11(B) shows the selectivity-time curves for the La-modified NiMoB catalysts. Doping of La increased the selectivity toward $p$-CAN. As shown earlier in the HRTEM image (Fig. 3), adding an appropriate amount of La could separate the NiMoB particles and prevent them from aggregating. During synthesis, both La and Mo were reduced by $\mathrm{NaBH}_{4}$ to form hydroxides in the aqueous solution. The presence of these hydroxides decreased the $\mathrm{NiB}$ particle size and made the Ni more unsaturated on the catalyst surface. The nitro group consists of two highly electronegative elements, $\mathrm{N}$ and $\mathrm{O}$. With electron donation from $\mathrm{B}^{0}, \mathrm{Mo}^{0}$, and $\mathrm{La}^{0}$ to $\mathrm{Ni}$, the $\mathrm{Ni}$ on the catalyst surface became electron-enriched. The highly electronegative $-\mathrm{NO}_{2}$ would therefore adsorb easily on the surface, and the active sites would activate and polarize the $\mathrm{N}=0$ bond. The $p a-$ $r a$-substituted nitro group has the highest electronegativity resulting from the combination of both inductive and resonance effects. For chlorine, only the inductive effect is present. Because the $-\mathrm{NO}_{2}$ group is more electronegative than $-\mathrm{Cl},-\mathrm{NO}_{2}$ is supposed to occupy the active $\mathrm{Ni}$ sites on the catalytic surface at the start of the reaction. During the reaction the $-\mathrm{NO}_{2}$ group adsorbed on the catalyst surface is hydrogenated to form $p$-CAN, which is further desorbed. When -Cl preoccupies the catalyst surface, hydrogenation followed by dehalogenation occurs, leading to the formation of nitrobenzene, which is eventually desorbed [17,37]. As a result of the chemical bonding in the nitro group, the $\mathrm{N}$ atom is positively charged, and each $\mathrm{O}$ atom has a partial negative charge. For this reason the nitro group strongly attracts electrons. The $-\mathrm{NH}_{2}$ of $p$-CAN might adsorb at the surface of alloyed $\mathrm{B}$ and coordinate with other $-\mathrm{NH}_{2}$ groups. Hence, this would improve the selectivity to $p$-CAN by depressing the dehalogenation reaction. La-dopants in the NiMoB catalysts further increase the electron density and serve to increase the selectivity.

The selectivities to $p$-CAN of various catalysts as the conversion of $p$-CNB reached $50 \%-100 \%$ are shown in Table 4 . The La-modified NiMoB catalysts exhibited higher selectivity to $p$-CAN than the unmodified catalyst. The selectivity of all La-doped samples exceeded $90 \%$. For the $0.2 \mathrm{La}-\mathrm{NiMoB}$ catalyst, the reaction reached completion within $60 \mathrm{~min}$, and the selectivity to $p$-CAN was $97.5 \%$. It is concluded that La-NiMoB is a good catalyst for hydrogenation of $p$-CNB. It is evident that the double-doped LaMo-NiB catalyst could further increase the dispersion of $\mathrm{NiB}$, which is beneficial for the hydrogenation reaction not only by enhancing the activity but also by increasing the selectivity to $p$-CAN.

The La-NiMoB sample was tested five times in succession. The activity decreased by $5 \%$ after the first run and was stable thereafter. The particle size was observed to increase slightly after the first run. The activity of the NiMoB sample decreased by $10 \%$ after the first run.

\subsection{Kinetics study}

The reaction rate constant for each catalyst was calculated. The conversion-time curves for the hydrogenation of $p$-CNB (Fig. 11) revealed that the reaction rate was first-order with respect to the concentration of $p$-CNB. The reaction rate constant $\left(r_{\mathrm{A}}\right)$ can be expressed as follows:

$$
-r_{\mathrm{A}}=k C_{\mathrm{A} 0} \cdot\left(1-X_{\mathrm{A}}\right)
$$

where $k$ is the reaction rate constant $\left(\mathrm{s}^{-1}\right), C_{\mathrm{A} 0}$ is the initial concentration of the reactant, and $X_{\mathrm{A}}$ is the extent of conversion. The reaction was carried out in a constant-volume batch reactor, therefore the rate equation becomes

$$
-r_{\mathrm{A}}=C_{\mathrm{A} 0} \mathrm{~d} X_{\mathrm{A}} / \mathrm{d} t
$$

Table 4

\begin{tabular}{|c|c|c|c|c|c|c|}
\hline \multirow{2}{*}{ Catalyst } & \multirow{2}{*}{$\begin{array}{c}\text { Rate constant } \\
\left(\mathrm{s}^{-1}\right)\end{array}$} & \multirow{2}{*}{$\begin{array}{l}\text { Time (min) at } \\
50 \% \text { conversion }\end{array}$} & \multirow{2}{*}{$\begin{array}{c}\text { Time (min) at } \\
100 \% \text { conversion }\end{array}$} & \multicolumn{3}{|c|}{ Selectivity (\%) } \\
\hline & & & & $p$-CAN & AN & NB \\
\hline NiMoB & 0.0231 & 49.3 & 88.3 & 93.0 & 7.0 & 0 \\
\hline 0.02La-NiMoB & 0.0543 & 14.0 & 64.2 & 95.3 & 4.7 & 0 \\
\hline 0.05La-NiMoB & 0.0476 & 18.8 & 66.2 & 94.8 & 5.2 & 0 \\
\hline 0.1La-NiMoB & 0.0363 & 27.5 & 60.2 & 94.4 & 5.6 & 0 \\
\hline 0.2La-NiMoB & 0.0699 & 9.7 & 58.1 & 97.5 & 2.5 & 0 \\
\hline 0.4La-NiMoB & 0.0380 & 14.4 & 80.7 & 97.8 & 2.2 & 0 \\
\hline 0.6La-NiMoB & 0.0395 & 9.9 & 86.2 & 96.2 & 3.8 & 0 \\
\hline 0.7La-NiMoB & 0.0308 & 22.2 & 119.2 & 97.2 & 2.8 & 0 \\
\hline 0.8La-NiMoB & 0.0295 & 27.3 & $>120$ & 98.6 & 1.4 & 0 \\
\hline
\end{tabular}

The effect of La content on the hydrogenation of $p$-CNB over La-NiMoB catalysts at $333 \mathrm{~K}$.

Reaction conditions: $\mathrm{H}_{2} 1.2 \mathrm{MPa}, 333 \mathrm{~K}$, absolute methanol solvent, $500 \mathrm{r} / \mathrm{min}$, Ni catalyst $0.2 \mathrm{mmol}$. 
where $t$ is the reaction time. Combining the two equations above using the integral method, one obtains

$$
-\ln \left(1-X_{\mathrm{A}}\right)=k t
$$

The reaction rate constant is therefore given as the gradient of the plot of $-\ln \left(1-X_{\mathrm{A}}\right)$ vs $t$. The results are shown in Table 4 . The rate constant of $0.2 \mathrm{La}-\mathrm{NiMoB}$ was $0.0699 \mathrm{~s}^{-1}$, which, as expected, was the highest among all samples.

\section{Conclusions}

A series of La-doped NiMoB nanoalloy catalysts with different La contents were prepared by chemical reduction using $\mathrm{NaBH}_{4}$ as the reducing agent. All La-NiMoB samples were amorphous, and the doping of La at low Li:Ni ratios decreased the degree of long-range order, enhanced the thermal stability, and decreased the particle size. However, high La:Ni ratios had the opposite effect. The shape of the La-NiMoB particles was spherical, and the particle size was in the range of 3-4 nm. In addition, adding La caused the binding energy of $\mathrm{Ni}^{0}$ to shift negatively, resulting in electron enrichment of $\mathrm{Ni}$. La not only donated electrons to $\mathrm{Ni}^{0}$ but also enhanced the electron donation from $\mathrm{B}^{0}$ and $\mathrm{Mo}^{0}$ to $\mathrm{Ni}^{0}$. Both lathanium and molybdenum hydroxides enhanced the dispersion of $\mathrm{NiB}$ and prevented it from aggregating. Because $-\mathrm{NO}_{2}$ is more electronegative than $-\mathrm{Cl}$, it is proposed that $-\mathrm{NO}_{2}$ occupied the active $\mathrm{Ni}$ sites on the catalytic surface at the start of the reaction. The $-\mathrm{NO}_{2}$ adsorbed at the catalyst surface was hydrogenated to form $p$-CAN, which subsequently desorbed. Therefore, the selectivity of the catalyst toward $p$-CAN increased with increasing La content. It should be noted that at high $\mathrm{Li}$ :Ni ratios, La would cover the Ni active sites, resulting in a decrease in activity. In addition, alloyed B activated the polar $-\mathrm{NO}_{2}$ group through the more electronegative $\mathrm{O}$ atom. The $-\mathrm{NH}_{2}$ of $p$-CAN might have adsorbed at the surface of the alloyed $\mathrm{B}$ and coordinated with other $-\mathrm{NH}_{2}$ groups, which may have improved the selectivity to $p$-CAN by depressing the dehalogenation reaction. 0.2La-NiMoB had the highest $\mathrm{Ni}^{0}$ and most electron-rich active sites, resulting in a higher activity and selectivity to the hydrogenation of $p$-CNB. The optimum molar ratio of La:Ni was 0.2 .

\section{Acknowledgements}

The authors would like to acknowledge Professor Yi Chen at
Nanjing University for inspiration on the research of metal boride. This work was supported by the Ministry of Economic Affairs of Taiwan.

\section{References}

[1] Liu Y C, Huang C Y, Chen Y W.J Nanopart Res, 2006, 8: 223

[2] Liu Y C, Huang C Y, Chen Y W. Ind Eng Chem Res, 2006, 45: 62

[3] Shen J Y, Hu Z, Zhang Q H, Zhang L F, Chen Y. J Appl Phys, 1992, 71: 5217

[4] Shen J H, Chen Y W. J Mol Catal A, 2007, 273: 265

[5] Shen J Y, Hu Z, Zhang L F, Li Z Y, Chen Y. Appl Phys Lett, 1991, 59: 3545

[6] Su J F, Zhao B, Chen Y W. Ind Eng Chem Res, 2011, 50: 1580

[7] Wang M H, Li H X, Wu Y D, Zhang J. Mater Lett, 2003, 57: 2954

[8] Chen X F, Li H X, Luo H S, Qiao M H. Appl Catal A, 2002, 233: 13

[9] Chen Y. Catal Today, 1998, 44: 3

[10] Chen L F, Chen Y W. Ind Eng Chem Res, 2006, 45: 8866

[11] Li H, Li H X, Dai W L, Wang W J, Fang Z G, Deng J F. Appl Surf Sci, 1999, 152: 25

[12] Li H X, Luo H S, Zhuang L, Dai W L, Qiao M H. J Mol Catal A, 2003, 203: 267

[13] Li H, Zhao Q F, Li H X.J Mol Catal A, 2008, 285: 29

[14] Li H X, Wu Y D, Zhang J, Dai W L, Qiao M H. Appl Catal A, 2004, 275: 199

[15] Li H, Zhang J, Li H X. Catal Commun, 2007, 8: 2212

[16] Wang W Y, Yang Y Q, Luo H A, Liu W Y. Catal Commun, 2010, 11: 803

[17] Zhao B, Chen Y W.J Non-Cryst Solids, 2010, 356: 839

[18] Liu Y C, Chen Y W. Ind Eng Chem Res, 2006, 45: 2973

[19] Ning J B, Xu J, Liu J, Miao H, Ma H, Chen C, Li X Q, Zhou L P, Yu W Q. Catal Commun, 2007, 8: 1763

[20] Yamashita H, Yoshikawa M, Funabiki T, Yoshida S. J Chem Soc, Faraday Trans I, 1986, 82: 1771

[21] Hou Y J, Wang Y Q, He F, Han S, Mi Z T, Wu W, Min E Z. Mater Lett, 2004, 58: 1267

[22] Fang J, Chen X Y, Liu B, Yan S R, Qiao M H, Li H X, He H Y, Fan K N. J Catal, 2005, 229: 97

[23] Deng J F, Li H X, Wang W J. Catal Today, 1999, 51: 113

[24] Dai W L, Qiao M H, Deng J F. Appl Surf Sci, 1997, 120: 119

[25] Hoelderich W F. Catal Today, 2000, 62: 115

[26] Okamoto Y, Nitta Y, Imanaka T, Teranishi S. J Chem Soc, Faraday Trans 1, 1979, 75: 2027

[27] Linderoth S, Mørup S. J Appl Phys, 1991, 69: 5256

[28] Lü L H, Ma Y H, Guo F, Ma R H, Xin J N, Wang Y, Kenji N. Fine Chem (吕连海, 马永欢, 郭方, 马荣华, 辛俊娜, 王越, 根岸贤治. 精细化

\section{Graphical Abstract}

Chin. J. Catal, 2013, 34: 2018-2028 doi: 10.1016/S1872-2067(12)60687-1

\section{Hydrogenation of $p$-chloronitrobenzene on La-doped NiMoB nanocluster catalysts}

Der-Shing Lee, Yu-Wen Chen*

National Central University, Taiwan, China

A series of La-Mo-doped, nanosized NiMoB catalysts with various La contents were prepared and characterized. Doping of La had a positive effect on the NiMoB catalyst properties and activity in the hydrogenation of $p$-chloronitrobenzene.

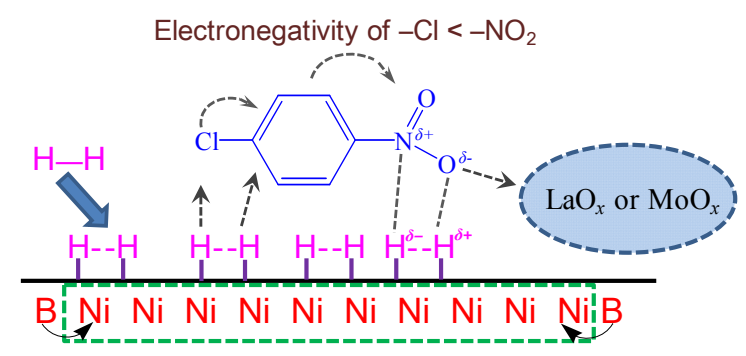


工), 2008, 25: 251

[29] Ma Y F, Li W, Zhang M H, Zhou Y, Tao K Y. Appl Catal A, 2003, 243 215

[30] Parks G L, Pease M L, Burns A W, Layman K A, Bussell M E, Wang X Q Hanson J, Rodriguez J A. J Catal, 2007, 246: 277

[31] Rajesh B, Sasirekha N, Lee S P, Kuo H Y, Chen Y W. J Mol Catal A, 2008, 289: 69

[32] Moulder J F, Stickle W F, Sobol P E, Bomben K D. Handbook of X-Ray Photoelectron Spectroscopy. Minnesota: Physical Electronics, Inc., 1995

[33] Xu Q, Liu X M, Chen J R, Li R X, Li X J. J Mol Catal A, 2006, 260: 299

[34] Yan X P, Liu M H, Liu H F, Liew K Y.J Mol Catal A, 2001, 169: 225
[35] Yan X H, Sun J Q, Wang Y W, Yang J F. J Mol Catal A, 2006, 252: 17

[36] Yan X H, Sun J Q Xu Y H, Yang J F. Chin J Catal (严新焕, 孙军庆, 徐 颖华, 杨建峰. 催化学报), 2006, 27: 119

[37] Zhao B, Chou C J, Chen Y W. Ind Eng Chem Res, 2010, 49: 1669

[38] Liu M H, Yu W Y, Liu H F, Zheng J M. J Colloid Interface Sci, 1999, 214: 231

[39] Han X X, Zhou R X, Lai G H, Zheng X M. Catal Today, 2004, 93-95: 433

[40] Han X X, Zhou R X, Zheng X M, Jiang H. J Mol Catal A, 2003, 193: 103

[41] Coq B, Tijani A, Figuéras F. J Mol Catal, 1992, 71: 317

[42] Coq B, Tijani A, Dutartre R, Figueras F. J Mol Catal, 1993, 79: 253

[43] Noller H, Lin W M.J Catal, 1984, 85: 25

\title{
对氯硝基苯在含镧的镍钼嗍奈米触媒上的氢化反应
}

\author{
李德馨, 陈郁文 ${ }^{*}$ \\ 中央大学化工与材料系, 台湾中㘯 320
}

\begin{abstract}
摘要: 钿和镧是好的镍嗍触媒的促进剂, 对于对氯硝基苯氢化反应有好的效果, 因此同时加入钿和锞在镍嗍触媒上应该是有趣的 研究题目. 本研究利用化学还原法制备了一系列含有不同量针的镧钿镍触媒, 醋酸镍、钼酸胺及硝酸镧同时加入含有甲醇的水溶 液中. 以X光绕射, 穿透式电子显微镜、高分辨率电子显微镜及X光光电能谱仪鉴定了这些触媒. 对氯硝基苯氢化反应是在批次反 应器中于 $333 \mathrm{~K} 、 1.2 \mathrm{MPa}$ 氢气压力及 $500 \mathrm{r} / \mathrm{min}$ 转速下操作. 即使在镍钼嗍中加入很少量的针, 对提高活性及对氯苯胺的选择性都 有显著的效果, 加入适量的针可以抑制镍钿嗍的长范围的结晶排列, 并保持镍锄嗍在非结晶状态. 针以氧化态存在, 其功效为阻 隔物, 避免镍钼硼聚集, 但是过量的镧会有相反的效果, 镧和镍的适当比例是 0.2 .
\end{abstract}

关键词: 硣化镍; 锞; 氢化反应; 含氯硝基苯; 含氯苯胺

收稿日期: 2013-03-30. 接受日期: 2013-06-05. 出版日期: 2013-11-20.

*通讯联系人. 电话: +886-3-4227151-34203; 传真: +886-3-4252296; 电子信箱: ywchen@ncu.edu.tw

本文的英文电子版由Elsevier出版社在ScienceDirect上出版(http://www.sciencedirect.com/science/journal/18722067). 